\section{Evidence for cost-effectiveness of ultrasound in evaluation of blunt trauma patients}

\author{
Bennett H. Lane \\ Harvard Medical School, Boston, MA, \\ USA
}

Abstract

The evaluation of the blunt trauma patient frequently includes the focused assessment with sonography for trauma (FAST) exam. Additional point-of-care applications for ultrasound in blunt trauma patients are emerging. Ultrasound is a noninvasive diagnostic technique generally considered to be relatively inexpensive, offering the potential for improved outcomes and lower costs. However, formal evaluation of ultrasound's effect on cost-effectiveness has occurred in a piecemeal fashion. The aim of this review is to evaluate the available evidence regarding whether ultrasound is cost-effective for evaluation of blunt trauma patients, and what factors may affect its cost-effectiveness.

\section{Introduction}

Since the early 1990s, clinical practice has shifted to incorporate the Focused Assessment with Sonography for Trauma (FAST) exam into the standard evaluation of the trauma patient. The paradigm shift of physicians at the pointof-care obtaining, interpreting, and acting on information in real-time using sonography, a non-invasive diagnostic technique generally considered to be relatively inexpensive, offered a new opportunity to improve care and lower cost. While the FAST exam and other applications of ultrasound (US) has become common over the last approximate quartercentury, evaluations of its effect on costs and outcomes has occurred in a piecemeal fashion.

\section{Clinical question}

This review of available evidence aimed to evaluate the question: Among emergency department patients presenting with blunt trauma, is ultrasound (US) cost-effective for evaluation and what factors affect cost-effectiveness?

\section{Materials and Methods}

Pubmed was searched for English-language articles published after May 23, 1996 using the following search phrases: cost effectiveness ultrasound trauma (136 results) and FAST exam ultrasound trauma (36 results). Of 171 results reviewed for relevance, 24 articles were selected for full text evaluation; of these, 18 were selected for inclusion. Handsearching reference lists in included articles generated 5 additional references. The Cochrane review database was also directly reviewed, but only a review previously identified by the Pubmed search strategy was identified. ${ }^{1}$ One reference selected for full text review was not available, ${ }^{2}$ but its data was included in the Cochrane review. This review focused on identifying sources helpful in evaluating a patient-level model of costs and outcomes that compared use of ultrasound to an alternative clinical scenario (i.e., no use of ultrasound). ${ }^{3}$ Notably, costs can be denominated in dollars and patient outcomes can be denominated in quality-adjusted life years (QALY), with the value of an intervention expressed as the incremental cost-utility ratio denominated in dollarsper-QALY; however, alternative measures for clinical outcomes (deaths prevented, strokes prevented) are also possible. ${ }^{3}$

\section{Costs of ultrasound evaluation in trauma patients}

Figure 1 summarizes a theoretical model for costs related to ultrasound (US). Costs for ultrasound include both fixed costs and variable costs. Examples of fixed costs include the cost of the machine and, if a dedicated room is used or if room occupancy is increased due to increased length-of-stay with ultrasound use, an allocation for a fixed building cost. Examples of variable costs include the costs associated with the operator's and image interpreter's time (in some cases, this may be the same person). A 3-year, single-center experience study of ultrasound use in surgical criti$\mathrm{cal} /$ intensive care units suggests the relative magnitude of costs, with costs for staff (mix of sonographer technician and surgeon time) roughly triple equipment-related costs. ${ }^{4}$

In cost-effectiveness analysis, differences in clinical course of patients when ultrasound is used compared to the alternative when ultrasound is not used must also be identified and estimated. The direction of such effects generally cannot be determined a priori. Empirical data about hypothesized changes in clinical course (e.g., decreased use of CT scans) are reviewed later in this paper.

\section{Primary literature addressing cost-effectiveness}

The literature search identified four high-
Correspondence: Bennett Lane, Harvard Medical School, Tosteson Medical Education Center, Rm 265A, 260 Longwood Avenue, Boston, MA, 02115, USA.

Tel: +1.617.416.6977 - Fax: +1.617.432.2500.

E-mail: Bennett_lane@hms.harvard.edu

Key words: Cost-effectiveness; Ultrasound; Trauma; FAST exam.

Acknowledgements: the author wishes to acknowledge the clerkship faculty of the Massachusetts General Hospital Emergency Medicine clerkship, Dr. James Takayesu and Dr. Emily Miller, for fostering his interest in this material.

Contributions: sole author responsible for all aspects of submission.

Conflict of interest: the author declares no potential conflict of interest.

Received for publication: 5 June 2016 .

Accepted for publication: 31 August 2016.

This work is licensed under a Creative Commons Attribution 4.0 License (by-nc 4.0).

(c) Copyright B.H. Lane, 2016

Licensee PAGEPress, Italy

Emergency Care Journal 2016; 12:6057

doi:10.4081/ecj.2016.6057

quality publications directly addressing costeffectiveness of ultrasound.

In 1999, Boulanger et al. published a prospective, single-center study of 706 blunt trauma patients with clinical suspicion for abdominal injury allocated to either a FAST or a no-FAST evaluation algorithm with randomization occurring at the trauma team leader level. The study reported differences in mean diagnostic costs (which appear similar to variable costs as described in Figure 1). ${ }^{5}$ Use of a FAST exam was less expensive (CA\$156) than no-FAST $(\mathrm{CA} \$ 540)(\mathrm{P}<0.0001)$, largely due to a much lower use of CT exam in the FAST group (24\%) compared to the no-FAST group (91\%). ${ }^{5}$ This study is limited by its single-center design, an alternative algorithm with greater use of DPL than is now common, and its failure to account for fixed costs. By ignoring fixed costs, the authors likely underestimated the magnitude of the cost benefit to the FAST group because (a) CT scanner machine acquisition costs are much higher than ultrasound machine costs and (b) the authors showed differences in mean time to diagnosis between the FAST group ( $53 \pm 55$ minutes) compared to the no-FAST group $(151 \pm 187$ minutes $)$ $(\mathrm{P}<0.0001)$ that would likely lead to higher room occupancy allocation costs. ${ }^{5}$

Melniker et al. conducted a prospective, twocenter randomized controlled trial that 
enrolled 262 torso trauma patients and had as the primary outcome variable time from ED arrival to transfer to operative care, which decreased with US by $64 \% .{ }^{6}$ Secondary endpoints included CT use [decreased with US, odds ratio (OR) 0.16], hospital length of stay (decreased with US by 27\%), and hospital charges (decreased with US by $35 \%$ in multiplicative change model). ${ }^{6}$ This study's strengths are its robust randomized design and clinically meaningful changes in outcomes. In evaluating cost-effectiveness, however, the study is dramatically limited by its use of hospital charges. Charges are taken from a hospital chargemaster and bear highly variable relationships to actual economic costs; recognizing this fact, most hospital financial transactions are completed based on hospital diagnosis-related groups (DRGs) or pre-specified private insurance contracts that do not refer to the chargemaster. In addition, the mechanism of the study's demonstrated decrease in costs is unclear with no specification of the relative contribution of decreased diagnostic testing (CT use), length of stay, and occurrence of complications (defined as hemorrhagic shock, septic shock, multisystem organ failure or death and which also decreased in US group with OR 0.17). ${ }^{6}$

Hall and colleagues performed a cost-effectiveness analysis of the cardiac component of the FAST exam using a decision tree model. ${ }^{7}$ Due to the rarity of blunt cardiac injury, the differences in QALYs between the FAST with cardiac component $v s$ without cardiac component were very small: 0.0003 QALYs in normotensive patients and 0.0065 QALYs in hypotensive patients; however, differences in cost were also estimated to be small (approximately $\$ 130$ in both populations), and the resulting incremental cost effectiveness ratios for inclusion of a cardiac component in the FAST exam were $\$ 19,918 /$ QALY for hypotensive patients and $\$ 465,867 /$ QALY for normotensive patients. ${ }^{7}$ Using either the UK NICE benchmark of $£ 20,000-£ 30,000 /$ QLLY or the United States benchmark standard popularized by David Cutler and others of $\$ 100,000 / \mathrm{QALY},{ }^{8}$ the use of a cardiac component in FAST is cost-effective in hypotensive patients but not in normotensive patients.

Kaye, et al, utilized a decision tree model to evaluate blunt cerebrovascular injury screening approaches, comparing costs and stroke rate between 5 screening approaches: no screening, duplex ultrasound, magnetic resonance angiography, [conventional] angiography, and CTA [CT angiography] in a high-risk blunt trauma population. ${ }^{9}$ For duplex ultrasound, relevant model inputs included sensitivity (0.400), specificity (0.999), and cost of ultrasound (\$333); the authors found that, on an institutional basis (i.e., ignoring the societal lifetime costs of stroke), duplex ultrasound screening was the most cost-effective at just $\$ 8,940$ per stroke prevented. ${ }^{9}$ CT angiography, which had higher model sensitivity (0.997) and costs (\$708), generated more strokes prevented at a marginally higher cost of $\$ 10,670$ per stroke prevented. ${ }^{9}$ Using a societal perspective, which assumes $\$ 608,483$ in lifetime stroke-associated costs, CTA was most costeffective (\$3727) and ultrasound was least cost-effective $(\$ 20,564)$ in a high-risk blunt trauma population. A limitation of this paper is that the authors chose to use cost of ultrasound alone, rather than incorporating a weighted estimate of the cost of follow-up CTA often used following ultrasound. Their finding that ultrasound was most cost-effective on an institutional basis is sensitive to this choice, and their sensitivity analysis does not delineate its effect.

In summary, the literature examining costs and outcomes of ultrasound evaluation of trauma patients is limited to relatively few studies examining costs specifically. The available studies do show cost-effectiveness for relatively specialized exams in at least some cases (the subxiphoid view of the FAST exam in hypotensive patients ${ }^{7}$ and carotid duplex US for blunt cerebrovascular injury ${ }^{9}$ ) and provide suggestive evidence for cost-effectiveness of abdominal ultrasound in blunt trauma.,

\section{Factors affecting cost-effec- tiveness of ultrasound evalua- tion of trauma patients}

Because medical decision-making is complex, a wide range of factors shape how ultrasound changes patient costs, clinical management and outcomes. Figure 2 outlines a simplified model for ultrasound's role in trauma patient evaluation and identifies four categories of factors likely to modulate its effect on either costs or outcomes (notably, this figure omits the possibility of incidental findings).

\section{Selection criteria}

One debate in the literature is whether a subset of blunt trauma patients should be selected for FAST while others are excluded (in favor of a routine CT protocol or other approach). The most commonly advanced criterion has been exclusion of hemodynamically stable (HDS) patients. Natarajan and colleagues have argued that given poor sensitivity of $41 \%$ in hemodynamically stable patients, the FAST exam should not be used to avoid CT scan use; furthermore, since FAST positive patients who were hemodynamically stable routinely receive a CT scan for better under-

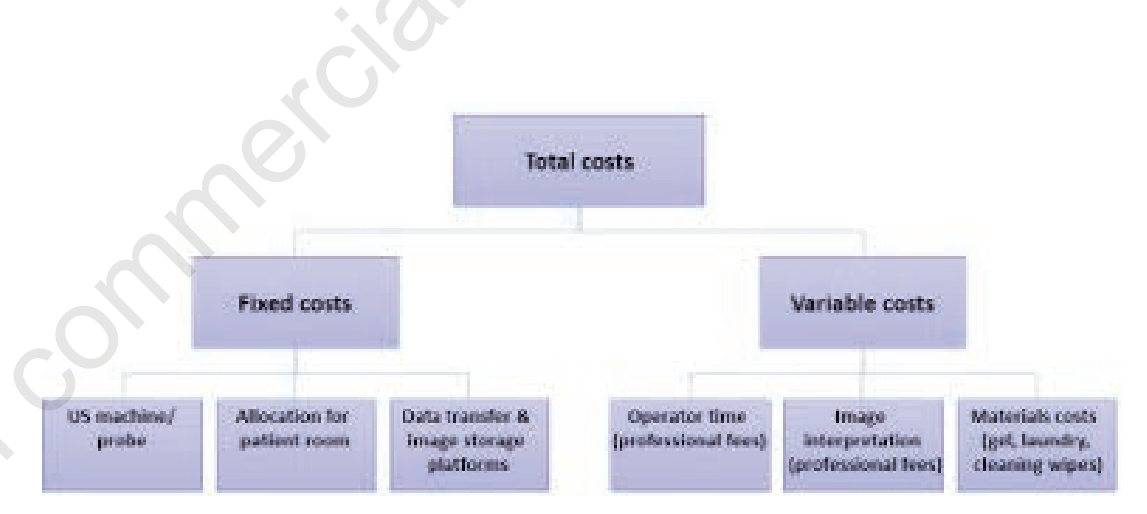

Figure 1. Model of costs of ultrasound in trauma patient assessment.

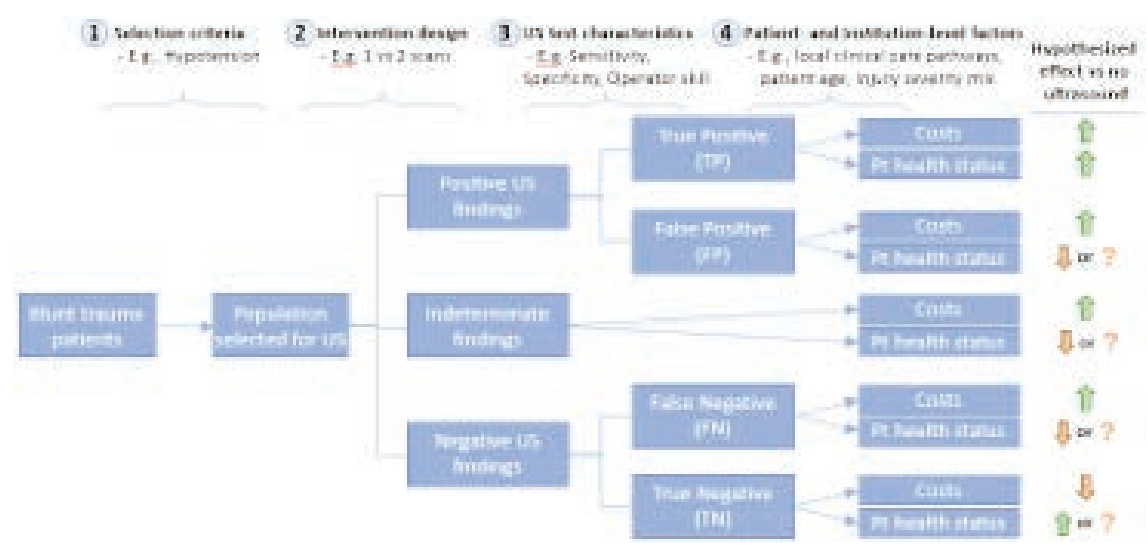

Figure 2. Model of determinants of cost-effectiveness. 
standing of the intraabdominal injuries and to decide on operative versus no-operative management the FAST exam could be omitted in stable patients entirely given no change in clinical management. ${ }^{10}$ Another study led by Miller and colleagues only examined hemodynamically stable patients and reported a comparable sensitivity of $42 \% .{ }^{11}$ However, other studies have found higher sensitivity rates (in part by including hemodynamically unstable patients who are more likely to have pathologic free fluid) and reported differences in the institutional-level choice to use CT routinely; due to these differences in institutional-level effects, the ability to generalize this recommendation to other sites is unclear.

\section{Intervention design}

While the FAST exam has essentially been standardized with 4 component views, institutional implementation of its use can vary. Blackbourne and colleagues examined secondary ultrasound, in which the FAST was repeated at a point after admission. ${ }^{12}$ In this prospective, observational, single-center study of 547 patients with initial and secondary US within 24 hours of admission (out of 1,361 with only an admission US and excluding patients who died, were discharged early, or had earlier operative intervention), the initial ultrasound had $31 \%$ sensitivity but sensitivity increased to $72 \%$ on secondary ultrasound. ${ }^{12}$ Specificity was $99.8 \%$ for both initial and secondary ultrasounds. ${ }^{12}$ Most notably, the NPV was $92 \%$ for initial US in the analyzed patient population and $96.6 \%$ with SUS. ${ }^{12}$ The notably improved NPV with secondary ultrasound suggests this approach could help address concerns about the poor sensitivity of ultrasound.

Another potential change in abdominal exams is the incorporation of contrast agents; while such agents remains investigational, commentators expect contrast agents can aid solid-organ injury detection. ${ }^{1,13}$

\section{Ultrasound test characteristics}

The utility of ultrasound will be greatest in improving patient outcomes and changing clinical management to minimize later costs if the test has good diagnostic performance; usually, this performance is described as sensitivity, specificity, PPV, and NPV (the latter two values are affected by the patient population). Several studies have attempted to characterize ultrasound's performance.

In 2001, Ma and colleagues published a 270 patient, prospective, 2-center non-randomized study in which the results of FAST were intentionally not used in clinical management; in this study, the FAST exam was found to be $89 \%$ sensitive and $99 \%$ specific. $^{14}$

In contrast, in 2005, Natarajan and colleagues published a much larger single-center retrospective registry study of 2,980 patients collected over 7 years, of whom 2130 had FAST examinations performed. ${ }^{9}$ Sensitivity was $43 \%$, specificity was $99 \%$, positive predictive value (PPV) was $95 \%$ and negative predictive value (NPV) was $94 \%$ for all blunt trauma patients and, in hemodynamically stable blunt trauma patients, sensitivity was $41 \%$ with similar other performance characteristics. ${ }^{10}$ Similar results were reported by Miller and colleagues in $2003,{ }^{11}$ although later commentators have noted some of the false negatives may have been clinically insignificant and that these studies reporting low-sensitivity have generally excluded unstable FAST patients. ${ }^{13}$

Sensitivity and specificity of the FAST exam are known to be significantly affected by operator experience. Ma and colleagues showed in a prospective, single-center, non-randomized study that emergency physicians with $<25$ post-training scans performed significantly worse than operators with $>50$ post-training scans [sensitivity 73 and 100\%, respectively, with non-overlapping $95 \%$ confidence intervals (CI) $].{ }^{15}$ Jang and colleagues showed that learning continues to improve beyond 50 scans with an error rate in consecutive scans 41-50 of $17 \%$ declining to $5 \%$ for scans $71-75$ in physicians learning FAST. ${ }^{16}$ Evidence suggests that skill improves until 200 scans, after which

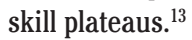

The presence of significant other injuries may also negatively affect the diagnostic performance of ultrasound. Becker and colleagues in a single-center retrospective registry study showed US had lower sensitivity (65\%) in patients with higher Injury Severity Scores (ISS $\geq 25$ ), as compared to ISS 16-24 (80\% sensitivity) or ISS $1-14$ ( $86 \%$ sensitivity).${ }^{17}$ Pelvic fractures or renal injuries were shown by Hoffman and colleagues to be associated with false negative FAST exams. ${ }^{18}$ In this singlecenter retrospective registry study with 1453 blunt abdominal trauma patients, of which 458 patients had both an US and confirmatory test within 12 hours, false negatives were significantly more likely with radiographically proven pelvic fracture (OR 3.46, 95\%CI 1.31-9.16) or operatively proven renal injury (OR 3.67, $95 \%$ CI 1.01-13.28). ${ }^{18}$

Studies also demonstrate impaired diagnostic performance of ultrasound in pediatric trauma patients compared to adult patients. In an expert opinion review, Rippey and Royse state that $31-37 \%$ of pediatric patients with an intra-abdominal solid-organ injury do not have hemoperitoneum compared to $22 \%$ of adults with injury; since US FAST exam detects free fluid, the FAST will be less sensitive for injury. ${ }^{13}$ Scaife and colleagues, in a single-center prospective study showed that in 128 patients with abdominal trauma who received an US, sensitivity for pathologic free fluid was $50 \%$, specificity was $85 \%$, PPV was $54 \%$, and NPV was $88 \% .^{19}$
Other factors affecting sensitivity that are referenced in the literature but without quantitative evaluation include other methodological differences in inclusion criteria or reference standard used, ${ }^{13}$ obese patients, ${ }^{10}$ presence of subcutaneous air, ${ }^{10}$ or equipment differences. $^{13}$

\section{Patient- and institution-level factors}

Critical to evaluating the role of ultrasound, but difficult to accurately assess and compare, is how clinicians use the information received from the test to alter management and, in turn, how patients' health status changes based on the care they receive. Three areas of clinical management effect have been studied extensively: the use of laparotomy, time to operative management, and CT scan utilization.

While laparotomy in an unstable patient with a positive FAST is considered clearly indicated, ${ }^{13}$ Moylan and colleagues found a positive FAST predicted a clinical requirement for laparotomy even in stable patients. ${ }^{20}$ In their retrospective two-center study of 1,636 normotensive $(\geq 100 \mathrm{mmHg})$ blunt trauma patients, they found a positive FAST had an unadjusted OR for therapeutic laparotomy of 116 (vs negative FAST) which persisted as an adjusted OR of 44.6 after controlling for a limited set of 7 confounding variables..$^{20}$ Among patients with a positive FAST exam, large fluid collections $(\geq 3 \mathrm{~cm})^{15}$ or fluid in the RUQ ${ }^{21}$ specifically have also been shown to predict a clinical a clinical course requiring exploratory laparotomy.

While measured in few studies, evidence is consistent that FAST can significantly reduce time from emergency department arrival to therapeutic laparotomy: Boulanger and colleagues found a reduction in mean time from 151 minutes to 53 minutes (Boulanger) while Melniker and colleagues reported change in median time from 157 minutes to 60 minutes with ultrasound use. ${ }^{4,5}$

Evidence suggests that use of FAST exams can decrease CT scans. A Cochrane review ( $\left.{ }^{1}\right)$ meta-analysis quantitatively synthesized 4 studies $^{2,5,6,22}$ and found CT scans were reduced by a risk differences estimate of -0.52 [95\% CI -0.82 to -0.21$]. .^{1}$ One of the included studies, a relatively small prospective randomized controlled trial with 208 enrolled patients at a single Level 2 trauma center with CT scan use as a primary endpoint showed significantly fewer patients required a CT scan after an ultrasound scan [ 36 vs $52 \%$, difference in proportions 15.9 (CI 2.6-29.1)]. ${ }^{22}$ In a prospective study in a single pediatric emergency department ( $n=128$ ultrasound scans of abdominal trauma patients) not included in the Cochrane review, surgeons indicated they would have cancelled CT scans in $48 \%$ of cases. ${ }^{19}$

The cost-effectiveness of ultrasound is also likely to be affected by institutional factors 
affecting cost. A central issue is that fixed costs associated with ultrasound (particularly the machine and data management costs) are minimized when the machine is used for many patients. In settings where FAST exams are less likely to be consistently used or to be used by relatively fewer patients, fixed costs may be a significant barrier. Pediatric settings, where the utility of FAST is less clear and a 2008 survey showed delayed uptake of the approach with only $15 \%$ of children's-only emergency departments indicating use of point-of-care ultrasound, ${ }^{23}$ are one such setting. Another is prehospital EMS where medical directors surveyed in 2014 most consistently identified cost of equipment as a barrier out of all factors included in the survey. ${ }^{24}$

\section{Conclusions}

Adoption of the FAST exam and other uses of ultrasound in evaluation of blunt trauma patient reflects clinicians' perception that the information obtained is helpful. In unstable patients, the FAST exam clearly aids diagnosis and reduces time to operative management. In stable patients, ultrasound's cost-effectiveness is unclear, but is likely to be greatest in adult patients with minor or moderate injury burden scanned by experienced operators whose clinical course is altered by results to enable decreased health care utilization and/or improved outcomes. An important mechanism for ultrasound's cost-effectiveness is its empirical effect of decreasing CT scan utilization, but at present no definitive study validates this use of ultrasound; this area represents an important avenue for future research.

\section{References}

1. Stengel D, Rademacher G, Ekkernkamp A, et al. Emergency ultrasound-based algorithms for diagnosing blunt abdominal trauma. Coch Data Syst Rev 2015;9: CD004446.

2. Arrillaga A, Graham R, York JW, Miller RS. Increased efficiency and cost-effective- ness in the evaluation of the blunt abdominal trauma patient with the use of ultrasound. Am Surg 1999;65:31-5.

3. Detsky AS, Naglie IG. A clinician's guide to cost-effectiveness analysis. Ann Intern Med 1990;113:147-54.

4. Murthi SB, Frankel HL, Narayan M, et al. Making the financial case for a surgeondirected critical care ultrasound program. J Trauma Acute Care Surg 2014;76:340-6.

5. Boulanger BR, McLellan BA, Brenneman FD, et al. Prospective evidence of the superiority of a sonography-based algorithm in the assessment of blunt abdominal injury. J Trauma 1999;47:632-7.

6. Melniker LA, Leibner E, McKenney MG, et al. Randomized controlled clinical trial of point-of-care, limited ultrasonography for trauma in the emergency department: the first sonography outcomes assessment program trial. Ann Emer Med 2006;48:22735.

7. Hall MK, Omer T, Moore CL, Taylor RA. Cost-effectiveness of the cardiac component of the Focused Assessment of Sonography in Trauma examination in blunt trauma. Acad Emer Med 2016;23:41523.

8. Phillips V. The value and limits of economic evaluation in policy analysis. Available from: http:/healthaffairs.org/blog/2015/09/ 01/the-value-and-limits-of-economic-evaluation-in-policy-analysis/

9. Kaye D, Brasel KJ, Neideen T, Weigelt JA. Screening for blunt cerebrovascular injuries is cost-effective. J Trauma 2011; 70:1051-7.

10. Natarajan B, Gupta PK, Cemaj S, et al. FAST scan: is it worth doing in hemodynamically stable blunt trauma patients? Surgery 2010;148:695-701.

11. Miller MT, Pasquale MD, Bromberg WJ, et al. Not so FAST. J Trauma 2003;54:52-60.

12. Blackbourne LH, Soffer D, McKenney M, et al. Secondary ultrasound examination increases the sensitivity of the FAST exam in blunt trauma. J Trauma 2004;57:934-8.

13. Rippey JCR, Royse AG. Ultrasound in trauma. Best Pract Res Clin Anaes 2009;23: 343-62.

14. Ma OJ, Gaddis G, Steele MT, et al. Prospective analysis of the effect of physician experience with the FAST examina- tion in reducing the use of CT scans. Emer Med Australasia 2005;17:24-30.

15. Ma OJ, Kefer MP, Stevison KF, Mateer JR. Operative versus nonoperative management of blunt abdominal trauma: role of ultrasound-measured intraperitoneal fluid levels. Am J Emer Med 2001;19:284-6.

16. Jang T, Kryder G, Sineff S, et al. The technical errors of physicians learning to perform focused assessment with sonography in trauma. Acad Emerg Med 2012;19:98101.

17. Becker A, Lin G, McKenney MG, et al. Is the FAST exam reliable in severely injured patients? Injury 2010;41:479-83.

18. Hoffman L, Pierce D, Puumala S. Clinical predictors of injuries not identified by focused abdominal sonogram for trauma (FAST) examinations. J Emerg Med 2009; $36: 271-9$.

19. Scaife ER, Rollins MD, Barnhart DC, et al. The role of focused abdominal sonography for trauma (FAST) in pediatric trauma evaluation. J Pediatric Surg. 2013;48:13771383.

20. Moylan M, Newgard CD, Ma OJ, et al. Association between a positive ED FAST examination and therapeutic laparotomy in normotensive blunt trauma patients. J Emerg Med 2007;33:265-71.

21. Rose JS, Richards JR, Battistella F, et al. The FAST is positive, now what? Derivation of a clinical decision rule to determine the need for therapeutic laparotomy in adults with blunt torso trauma and a positive trauma ultrasound. J Emerg Med 2005;29:15-21.

22. Rose JS, Levitt A, Porter J, et al. Does the presence of ultrasound really affect computed tomographic scan use? A prospective randomized trials of ultrasound in trauma. J Trauma 2001;51:545-50.

23. Scaife ER, Fenton SJ, Hansen KW, Metzger RR. Use of focused abdominal sonography for trauma at pediatric and adult trauma centers: a survey. J Pediatric Surg 2009;44:1746-9.

24. Taylor J, McLaughlin K, McRae A, et al. Use of prehospital ultrasound in North America: a survey of emergency medical services medical directors. BMC Emerg Med 2014;14:6. 